\section{Ridge: A program to perform ridge regression analysis}

\section{ANDREW J. BUSH \\ Memphis State University, Memphis, Tennessee 38152}

Following development of the theoretical basis for ridge regression by Hoerl and Kennard (1970), the technique has enjoyed increasing popularity as a strategy for providing estimates of regression coefficients with a smaller total mean square error than their ordinary least-squares (OLS) counterparts (Price, 1977). From a computational point of view, the ridge estimates are calculated in the same manner as OLS coefficients, except that the principal diagonal of the predictor correlation matrix is incremented by a small positive value prior to inversion. Unfortunately, selection of the appropriate increment is not automatic.

Although ridge estimates can be computed by OIS programs using data transgeneration procedures, output from such programs is deficient in many important respects, including incorrect values for squared multiple correlations (SMCs), erroneous standard errors of estimate, and omission of indices that assist investigators in choosing acceptable ridge coefficients (cf. Darlington, 1978). The program described here overcomes such problems by returning correct values for regression statistics and providing estimates for selecting increments for the predictor correlation matrix as offered by Dempster, Schatzoff, and Wermuth (1977), Hoerl, Kennard, and Baldwin (1975), Kasarda and Shih (1977), Lawless and Wang (1976), and McDonald and Galarneau (1975). In conjunction with use of the ridge trace as suggested by Hoerl and Kennard (1970), these estimates of appropriate ridge increments can offer collaborative evidence for favoring one ridge solution (or range of solutions) over others.

Description. The main program uses a set of FORTRAN IV subroutines to produce statistical estimates and complete all auxiliary data manipulations. Nearly all work is done using singly dimensioned arrays.

Subroutine RCALC produces Pearson productmoment correlation coefficients, standard deviations, and variable means for raw input data. If residuals are requested, RCALC also writes the input data to a scratch file $(F: 8)$ for later access. Subroutine HOW is a modified version of the routine published by Cooley and Lohnes (1971). It is used to compute principal components and eigen values of the predictor correlation matrix and to estimate component regression weights, structures, and validities. CHOLESKY performs a Cholesky factorization on the predictor correlation matrix and identifies linear dependencies among predictors. INVCHO inverts the Cholesky factor and INVMUL produces the inverse of the predictor correla- tion matrix through operations on the inverted Cholesky factor. Subroutine CALBETA computes standardized regression weights, partial correlations, semipartial correlations, and partial $F$ ratios. It also computes the covariance and correlation of predictors with residuals. COVAR computes the regression weight variancecovariance matrix. REOUT computes predicted scores and residuals. VPRNT provides matrix printouts, SUMMAR writes summary output to a scratch file $(F: 9)$, and PUMP provides a copy of all summary out put at the program's conclusion.

Input. The job deck contains the following cards. Cards 1-3 are for user title and comments. Card 4 specifies the total number of variables to be included in analysis and the number of observations on the dependent variable in I2, 13 format. Card 5 specifies input data type and output options in $3 \mathrm{~A} 4$ format. The data type is RAW, FULL, or TRI for raw data, complete correlation matrix, or lower triangular correlation matrix input, respectively. The first output option controls summary displays and is coded either YES or ONLY or left blank for requesting, respectively, summary output in addition to regular output, summary output only, or no summary output. The second input option controls printing of residuals. By coding YES, residuals will be computed and displayed if the input data form is RAW. All entries on Card 5 are left-justified within fields. Card 6 specifies the data format, with the dependent variable specified last. Card 7 and subsequent cards are reserved for data according to user-specified type and format. Following the data cards, an analysis control card is required. For OLS analysis only on the data, the analysis control card is coded STOP; for further analyses on other data sets, the card is coded YES and the entire job deck is repeated for new input For ridge regression, the analysis control card content is RIDG and is followed by a terminal card that specifies the value to be used in successively incrementing the diagonal of the predictor correlation matrix, the maximum increment to be employed, and the starting increment in 3 F 10.4 format.

Output. The printed output includes user-prepared titling information followed by analysis results. If the output is not restricted to summary information, it will incorporate: (1) principal component regression results, including the eigen values and eigen vectors of the predictor correlation matrix, the principal components structure matrix, each component's regression weight, and each component's correlation with the dependent variable; (2) the input correlation matrix and the inverse of the predictor correlation matrix; (3) a series of regression calculations, including the current ridge increment, the determinant of the predictor correlation matrix, the current multiple correlation and SMC, the coefficient of alienation, the residual variance, predicted 
score variance, the sum of squared regression weights, and three estimates of appropriate ridge increments (those proposed by Hoerl et al., 1975; Lawless \& Wang, 1976; McDonald \& Galarneau, 1975); (4) the matrix of regression weight variance-covariances followed by the trace of the matrix, an estimate of squared bias, and an estimate of the expected total mean square error of the weights; (5) a listing of each predictor's validity, standardized weight, semipartial and partial correlation with the dependent variable, standard error of estimate, structure, and partial F ratio; and (6) observed dependent variable values, predicted values, and the set of residuals. For raw input, variable means, standard deviations, and unstandardized regression weights are also printed.

If ridge regression is specified, the majority of output elements listed above are displayed for each trial value of the ridge increment. However, for any increment greater than zero, $\mathrm{F}$ ratios are omitted and the covariance and correlation of predictors with error are substituted for partial and semipartial correlation listings. In addition, the summary output provides the SMC, the sum of eigen value reciprocals, the estimated total mean square error of the weights for use with the Kasarda and Shih (1977) criterion, the sum of squared regression weights for use with the McDonald and Galarneau (1975) criterion, an index of closeness of approach to the Dempster et al. (1977) criterion, and the regression coefficients corresponding to each ridge increment.

If, during the regression analysis, a linear dependency is encountered, the program isolates the dependency and reports its location prior to aborting the run.

Restrictions. The data matrix may not have more than 49 predictors. Since most problems have far fewer, this is not a major restriction. At present, the total number of observations is limited to 999 . This limit can be easily increased by altering the appropriate input format statement from I3 to I4. Since raw data are not stored internally, no other changes are required for reading larger data sets.

Computer and Language. The program is written in standard FORTRAN IV and has been tested on the
Memphis State University Xerox Sigma 9 computer. No reprogramming should be required for conversion to different computing systems, other than replacing the Xerox standard data reference numbers, F:105 and F: 108 , with those of the system to which the program is being adapted. For ease of conversion, all data reference numbers are identified and assigned early in the source. The program is well documented with comments and executes easily in timesharing modes with access to $25 \mathrm{~K}$ words or more.

Availability. The listing of the source program is available, at a charge of $\$ 1$ to cover postage and handling, from the author, Andrew J. Bush, Foundations of Education, Memphis State University, Memphis, Tennessee 38152.

\section{REFERENCES}

Cooley, W. W., \& Lohnes, P. R. Multivariate data analysis. New York: Wiley, 1971.

Darlington, R. B. Reduced variance regression. Psychological Bulletin, 1978, 85, 1238-1255.

Dempster, A. P., Schatzoff, M., \& Wermuth, N. A simulation study of alternatives to least squares. Journal of the American Statistical Association, 1977, 72, 77-91.

Hoe Rl, A. E., \& Kennard, R. W. Ridge regression: Biased estimation for nonorthogonal problems. Technometrics, 1970, 12, 55-67.

Hoerl, A. E., Kennard, R. W., \& Baldwin, K. F. Ridge regression: Some simulations. Communications in Statistics, $1975,4,105-123$

Kasarda, J. D., \& Shin, W. P. Optimal bias in ridge regression approaches to multicollinearity. Sociological Methods and Research, 1977, 5, 461-470.

LaWless, J. F., \& WANG, P. A simulation study of ridge and other regression estimators. Communications in statistics: Theory and methods, 1976, A5 (4), 307-323.

McDonald, G. C., \& Galarneau, D. I. A Monte Carlo evaluation of some ridge-type estimators. Journal of the American Statistical Association, 1975, 70, 407-416.

PrICE, B. Ridge regression: Application to nonexperimental data. Psychological Bulletin, 1977, 84, 759-766.

(Received for publication January 23, 1980; accepted January 24,1980 .) 